\title{
Efecto de la suplementación estratégica en un sistema de lechería en Cundinamarca, Colombia
}

\author{
Strategic supplementation effect in a dairy system in Cundinamarca, Colombia \\ Sergio Gómez-Vega ${ }^{1}$, Ronald Caicedo-Pinzón ${ }^{1}$, Juan Vargas-Martínez ${ }^{2,3}$
}

\section{Resumen}

El estudio tuvo como objetivo evaluar el efecto de la suplementación estratégica sobre la respuesta productiva y económica de un sistema de lechería en Cundinamarca, Colombia. Se seleccionaron seis vacas lactantes, de $455 \pm 65 \mathrm{~kg}$ de peso vivo, $100 \pm 4.94$ días en leche y $10 \pm 0.2 \mathrm{~L}$ de producción diaria de leche. Los animales fueron distribuidos al azar en dos esquemas de suplementación: comercial (forraje + suplemento comercial) y estratégico (forraje + suplemento estratégico). Se determinó la producción y composición láctea, el consumo de alimento y la condición corporal durante tres periodos de 20 días. Además, se determinaron los costos de producción e indicadores económicos. Las variables productivas fueron analizadas a través de un modelo de sobre cambio compuesto, mientras que las económicas a través de bloques completos al azar. Los resultados sugieren que la suplementación estratégica no modificó el consumo de alimento, la producción de leche, la composición láctea, ni los indicadores económicos; sin embargo, el costo del suplemento estratégico fue $37.5 \%$ menor que el suplemento comercial, lo que podría permitir reducir los costos de alimentación sin afectar los parámetros productivos en un sistema de producción lechero.

Palabras clave: calidad de leche; costos de producción; producción animal; sistemas pastoriles

\footnotetext{
${ }^{1}$ Semillero de Investigación en Nutrición Animal, Facultad de Ciencias Agropecuarias, Universidad de Ciencia Aplicadas y Ambientales, UDCA, Bogotá, Colombia

${ }^{2}$ Grupo de Investigación en Microbiología y Nutrición Animal del Trópico, Red de Ganadería y Especies Menores, Corporación Colombiana de Investigación Agropecuaria (Agrosavia), Bogotá, Colombia

${ }^{3}$ E-mail:jvargasm@agrosavia.co
}

Recibido: 4 de octubre de 2018

Aceptado para publicación: 30 de abril de 2019 
The aim of this study was to evaluate the effect of strategic supplementation on the productive and economic response of a dairy system in Cundinamarca, Colombia. Six lactating cows were selected. Animals were $455 \pm 65 \mathrm{~kg}$ body weight, $100 \pm 4.94$ days in milk and $10 \pm 0.2 \mathrm{~L}$ of daily milk production. Cows were randomly distributed in two supplementation schemes: commercial (forage + commercial supplement) and strategic (forage + strategic supplement). Dairy production and composition, feed intake and body condition were determined during three periods of 20 days each. In addition, production costs and economic indicators were determined. The productive variables were analyzed through a switch back model, while the economic ones through randomized complete blocks. The results suggest that strategic supplementation did not modify feed intake, milk production, milk composition, or economic indicators. However, the costs of the supplement decreased by $37.5 \%$. In conclusion, the strategic supplementation would reduce feed costs without affecting productive parameters in a dairy production system.

Key words: milk quality; production costs; animal production; grasslands systems

\section{INTRODUCCIÓN}

Los sistemas de producción animal requieren la generación de prácticas que promuevan la sostenibilidad, mejoren la calidad de vida del productor rural, cubran la demanda de proteína de origen animal y disminuyan el impacto sobre el ambiente (Makkar, 2016). Los rumiantes no son ajenos a esta realidad, y es por esto que algunos autores sugieren que la intensificación sostenible se configura como un mecanismo que podría dar solución a los retos que enfrenta la producción animal (Rao et al., 2015).

La literatura indica que el mejoramiento de los sistemas de alimentación, a través del suministro de una dieta balanceada, permite incrementar la producción animal, disminuir la excreción de nutrientes, mejorar el estatus sanitario e incrementar los ingresos del productor (Garg et al., 2013). Los costos de alimentación representan el $40 \%$ de los costos de producción en los sistemas ganaderos en trópico alto colombiano (Fedegan-SENA, 2013). En este sentido, la generación de estrategias que permitan disminuir este rubro permitiría impactar positivamente en la rentabilidad de los sistemas de producción, resultando en el aumento del ingreso y el mejoramiento en la calidad de vida del productor.

La suplementación con concentrados comerciales es una actividad que se realiza de manera tradicional en los sistemas de lechería especializada (Pulido, 2005). Sin embargo, estos productos son balanceados de acuerdo a los requerimientos nutricionales y la composición química promedio de una población animal y de los recursos forrajeros presentes en los sistemas de producción. Esta aproximación global limita el balanceo adecuado de nutrientes para contextos específi$\cos$ de producción. En este sentido, la nutrición de precisión requiere la incorporación de prácticas de suplementación estratégica que tenga en cuenta las características particulares de cada predio (requerimientos nutricionales de los animales y aportes de nutrientes de los recursos presentes en el predio) con miras a optimizar la respuesta productiva (Avellaneda et al., 2018). 
De esta manera, la suplementación estratégica es considerada una actividad que promueve sistemas ganaderos sostenibles debido a que: i. Potencializa la respuesta animal; ii. Reduce la excreción de nutrientes al ambiente; iii. Disminuye los costos de suplementación; y iv. Aumenta la rentabilidad de los sistemas de producción. Sin embargo, pocos trabajos han evaluado económicamente la incorporaión de suplementos estratégicos en la dieta de rumiantes (Avellaneda et al., 2018). El objetivo del presente trabajo fue evaluar el efecto de la suplementación estratégica sobre los parámetros productivos y económicos en un sistema de lechería especializada en trópico alto colombiano.

\section{Materiales y Métodos}

\section{Localización}

El trabajo experimental se desarrolló entre junio y agosto de 2017, en un predio ubicado en la vereda Yayatá, localizada en latitud norte $5^{\circ} 10^{\prime} 16.6^{\prime \prime}$ y longitud oeste 747'4.6", en el municipio de Pacho, Cundinamarca, Colombia. La región presenta una distribución bimodal de las lluvias con precipitación media de $1670 \mathrm{~mm}$ anual y temperatura promedio de $19{ }^{\circ} \mathrm{C}$ (IDEAM, 2018).

\section{Dietas Experimentales}

El manejo y el diseño experimental fue aprobado por el Comité de Carrera de Zootecnia de la Facultad de Ciencias Agropecuarias de la Universidad de Ciencias Aplicadas y Ambientales. Se utilizaron seis vacas mestizas lactantes, con $455 \pm 65 \mathrm{~kg}$ de peso vivo promedio, $100 \pm 4.94$ días en leche y $10 \pm 0.24$ litros de producción diaria de leche. Los animales fueron distribuidos en dos grupos al azar: manejo comercial (forraje + suplemento comercial) y suplemento estratégico formulado de acuerdo con la composición química del forraje y los requerimientos nutricionales de los animales (forraje + suplemento estratégico) (NRC, 2001). El su- plemento estratégico fue elaborado con materias primas disponibles en la región y elaborado en el predio (Cuadro 1). El suplemento comercial fue obtenido de una empresa agropecuaria local.

El experimento tuvo una duración de 60 días, distribuidos en tres periodos de 20 días cada uno. Los primeros 15 días de cada periodo se consideraron de acostumbramiento al suplemento (comercial o estratégico), mientras que los cinco días finales fueron considerados de medición. Los animales estuvieron en pastoreo en un mismo grupo durante toda la fase de experimen-tación. La base forrajera fue kikuyo (Cenchrus clandestinus) y se ofreció grupalmente de acuerdo al manejo tradicional del predio. Los suplementos fueron ofrecidos individalmente en el momento del ordeño de la mañana. Los animales tuvieron acceso ad libitum al agua.

En los días de evaluación de cada periodo se determinó el consumo de materia seca como la suma del consumo de suplemento y de forraje, determinado este último por el método agronómico (Valencia, 2011). Así mismo, la producción y la composición láctea se determinó diariamente y de manera individual durante cada periodo de medición. La condición corporal (Macdonald y Roche, 2011) y el peso vivo, a través de cinta bovino-métrica, fueron evaluados al inicio y al final de cada periodo. Además, se tomaron muestras diarias de los forrajes y los suplementos, las mismas que fueron pesadas (Navigator ${ }^{\circledR}$ XL, $10 \pm 0,001 \mathrm{~kg}$, Ohaus ${ }^{\circledR}$ ), mezcladas por periodos de muestreo y conservadas para su posterior análisis.

La evaluación económica de la suplementación se realizó a través del análisis de costos parciales descrito por Perea et al. (2018). Brevemente, se estimó el índice de conversión económico (ICE) como el producto entre índice de conversión alimenticia (ICA) y el precio de la dieta. El beneficio neto $(\mathrm{BN})$ se determinó como la diferencia entre los ingresos por la venta de leche y los costos incurridos en la alimentación (Cuadro 2).

\section{Análisis de Laboratorio}


Cuadro 1. Composición química, proporción de ingredientes y costo de las dietas experimentales

\begin{tabular}{|c|c|c|c|}
\hline Característica & Pasto kikuyo & $\begin{array}{c}\text { Balanceado } \\
\text { comercial (\%) }\end{array}$ & $\begin{array}{c}\text { Balanceado } \\
\text { estratégico (\%) }\end{array}$ \\
\hline \multicolumn{4}{|l|}{ Ingredientes } \\
\hline Maíz & & & 4.7 \\
\hline Salvado de trigo & & & 31.6 \\
\hline Glicerol & & & 25.0 \\
\hline Melaza & & & 13.4 \\
\hline Palmiste & & & 25.3 \\
\hline \multicolumn{4}{|l|}{ Nutrientes } \\
\hline Materia seca & 20.4 & 88.0 & 85.3 \\
\hline Proteína cruda & 13.0 & 21.2 & 12.0 \\
\hline Fibra detergente neutro & 73.4 & 38.7 & 39.9 \\
\hline Fibra detergente ácido & 32.8 & 15.2 & 16.4 \\
\hline Extracto etéreo & 1.5 & 8.3 & 3.1 \\
\hline Cenizas & 9.1 & 6.5 & 6.9 \\
\hline Materia orgánica & 90.9 & 93.7 & 93.1 \\
\hline Contenido celular & 26.6 & 61.2 & 60.1 \\
\hline Degradabilidad $^{1}$ & 44.06 & 66.36 & 78.68 \\
\hline Costo $\left(\$ C O L \mathrm{~kg} \mathrm{MS}^{-1}\right)^{*}$ & 230.60 & $1,306.50$ & 816.70 \\
\hline
\end{tabular}

En el forraje y en los suplementos comercial y estratégico se determinaron la concentración de materia seca (MS, AOAC, 2005), proteína cruda (PC, AOAC, 2005), cenizas (AOAC, 2005), extracto etéreo (EE, AOAC, 2005), fibra detergente neutro (FDN) y fibra detergente ácida (FDA, Van Soest et al., 1991) y la digestibilidad in vitro de la materia seca a las $48 \mathrm{~h}$ (Tilley y Terry, 1963). Además, se estimó la concentración de materia orgánica y contenido celular (Vargas et $a l ., 2014)$. La concentración individual de grasa (AOAC, 2005) y proteína (AOAC, 2005) en la leche se determinó en muestras colectadas diariamente.

\section{Diseño Experimental}

Las variables de respuesta animal fueron evaluadas a través de un modelo de sobrecambio compuesto con dos tratamientos (comercial y suplementación estratégica) aplicados en secuencia a través de tres periodos de evaluación (Martínez et al., 2011). 
Para las variables económicas se empleó un análisis de bloques completos al azar, en donde el factor del bloqueo fue el periodo. Para los análisis de varianza se utilizó el procedimiento GLM de SAS ${ }^{\circledR}(2017)$ de acuerdo con lo descrito por Martínez et al. (2011). Las medias fueron comparadas por medio de la prueba de Tukey con una significancia del $5 \%$.

\section{Resultados y Discusión}

\section{Respuesta Productiva}

El suplemento estratégico presentó una concentración menor de proteína y una degradabilidad mayor de la materia seca respecto al suplemento convencional (Cuadro 1). Algunos autores sugieren que los sistemas de alimentación en el trópico alto colombiano requieren la suplementación de recursos energéticos y con bajos tenores de proteína (glicerol, melaza), debido a que el kikuyo, principal pastura en este tipo de sistemas, se caracteriza por bajos niveles de carbohidratos no estructurales y elevadas concentraciones de nitrógeno (Correa et al., 2008; Vargas et al., 2018). En este sentido, la suplementación estratégica con mezclas bajas en proteína permitirían disminuir el costo de la suplementación y la excreción de nitrógeno en leche (Avellaneda et al., 2018), lo que se asocia con un mejor balance en la fermentación ruminal, menor gasto energético en la eliminación de urea y reducción en las tasas de excreción de nitratos y de producción de óxido nitroso.

No hubo diferencia estadística entre tratamientos con relación a la producción y composición de la leche (Cuadro 2). La literatura reporta que la inclusión de materias primas energéticas en la dieta de bovinos genera una mayor producción de leche cuando existe una limitada capacidad de consumo de materia seca, especialmente cuando el forraje es de baja calidad y puede limitar el consumo de alimento (Álvarez et al., 2007); sin embargo, otros trabajos no reportan diferencias al incluir suplementos balanceados en dietas soportadas en forrajes (Roche et al., 2017).

La ingestión de nutrientes está regulada por factores intrísecos y extrísecos del animal (Tarazona et al., 2012). En este sentido, dietas que presentan altos niveles de fibra podrían limitar la capacidad de consumo, mientras que dietas con baja calidad nutricional limitan la disponibilidad de nutrientes. En este trabajo, la ausencia de diferencias en la respuesta productiva, tanto en la condición corporal como en la relación entre el consumo de materia seca y la producción de leche entre tratamientos, podría explicarse por el consumo similar de forraje y de suplemento (Cuadro 2). Esto sugiere que las digestibilidades in vivo fueron similares entre las dietas a pesar que la degradabilidad in vitro de los materiales fue mayor en el suplemento estratégico respecto al convencional (Cuadro1).

La evaluación de la degradabilidad in vitro se ha relacionado con la digestibilidad in vivo de la materia seca (Tilley y Terry, 1963); sin embargo, algunos autores sugieren que los métodos in vitro estáticos no consideran variables tales como la selectividad, las tasas de paso diferencial del alimento y del fluido ruminal y la secreción salivar, entre otros (López, 2005). Es por esto que las técnicas in vitro, si bien permiten la evaluación inicial de un gran número de muestras, posteriormente se debe realizar la validación en modelos animales tendientes a identificar la respuesta productiva del individuo.

\section{Respuesta Económica}

Los animales que recibieron la suplementación estratégica presentaron menores costos de alimentación respecto a los que recibieron la suplementación comercial $(\mathrm{p}<0.05$; Cuadro 3), debido a una disminución del $37.5 \%$ de los costos en la elaboración del suplemento estratégico (Cuadro 1), pese a que el consumo se incrementó en un 
Cuadro 2. Consumo de alimento y respuesta productiva de vacas suplementadas con suplemento comercial o estratégico ${ }^{1}$

\begin{tabular}{|c|c|c|c|c|}
\hline Variable & Convencional & Estratégico & SEM $^{2}$ & $\mathrm{p}$ \\
\hline Condición corporal (kg) & 4.5 & 4.4 & 0.02 & $\mathrm{~ns}$ \\
\hline Peso vivo & 439.8 & 471.9 & 1.81 & ns \\
\hline \multicolumn{5}{|l|}{ Producción láctea $\left(\mathrm{kg} \mathrm{d}^{-1}\right)$} \\
\hline $\mathrm{LCG}^{3}$ & 13.6 & 14.5 & 0.30 & ns \\
\hline Grasa & 0.59 & 0.64 & 0.02 & ns \\
\hline Proteina & 0.39 & 0.40 & 0.02 & ns \\
\hline \multicolumn{5}{|l|}{ Composición láctea (\%) } \\
\hline Grasa & 4.9 & 5.2 & 0.19 & ns \\
\hline Proteina & 3.4 & 3.4 & 0.03 & ns \\
\hline \multicolumn{5}{|l|}{ Consumo (kg MS d $\left.{ }^{-1}\right)$} \\
\hline Forraje & 18.6 & 18.6 & - & - \\
\hline Suplemento & 2.3 & 2.5 & 0.01 & ns \\
\hline Total & 20.9 & 21.1 & 0.01 & ns \\
\hline $\begin{array}{l}\text { Consumo MS }(\mathrm{kg}) \text { / producción } \\
\text { de leche }(\mathrm{kg})\end{array}$ & 1.92 & 1.87 & 0.01 & ns \\
\hline \multicolumn{5}{|c|}{$\begin{array}{l}{ }^{1} \text { Convencional (suplemento comercial); Estratégico: formulado de acuerdo con la composición quír } \\
\text { del forraje y los requerimientos nutricionales de los animales } \\
{ }^{2} \text { Error estándar } \\
{ }^{3} \text { LCG: Leche corregida grasa al } 4 \% \\
\text { ns: no significativo }\end{array}$} \\
\hline
\end{tabular}

Cuadro 3. Consumo, costos e ingresos en el grupo de vacas con suplemento comercial o estratégico

\begin{tabular}{|c|c|c|c|}
\hline Variable & Convencional & Estratégico & SEM \\
\hline \multicolumn{4}{|c|}{ Consumo $\left(\mathrm{kg} \mathrm{MS} \mathrm{grupo}{ }^{-1}\right.$ periodo $\left.^{-1}\right)$} \\
\hline Forraje & 279.3 & 279.3 & - \\
\hline Suplemento & 34.8 & 35.8 & 0.7 \\
\hline Total & 314.1 & 314.8 & 6.3 \\
\hline \multicolumn{4}{|c|}{ Costos $\left(\$ C O L\right.$ grupo $^{-1}$ periodo $\left.^{-1}\right)$} \\
\hline Forraje & $30,720.0$ & $30,720.0$ & - \\
\hline Suplemento & $45,521^{\mathrm{a}}$ & $28,999^{\mathrm{b}}$ & 938.1 \\
\hline Total & $76,241^{\mathrm{a}}$ & $59,718^{\mathrm{b}}$ & 1699.5 \\
\hline \multicolumn{4}{|c|}{ Ingresos $\left(\$ C O L\right.$ grupo $^{-1}$ periodo $\left.^{-1}\right)$} \\
\hline Producción láctea & $174,828.0$ & $187,017.0$ & $10,666.1$ \\
\hline \multicolumn{4}{|l|}{ Indices $^{1}(\%)$} \\
\hline ICA & 1.61 & 1.46 & 0.08 \\
\hline ICE & $24,272.0$ & $17,477.0$ & $1,068.7$ \\
\hline $\mathrm{BN}$ & $98,587.0$ & $127,299.0$ & $1,0076.0$ \\
\hline
\end{tabular}

a.b Superíndices diferentes dentro de la misma fila representa diferencias significativas $(p<0.05)$

${ }^{1}$ ICA: Indice de conversión alimenticia; ICE: Indice de conversión económico; BN: beneficio neto 
leve 3\% (Cuadro 3). Sin embargo, como el consumo de suplemento representaba el $11.1 \%$ del consumo de materia seca total, el beneficio neto no se modificó significativamente, a pesar que el grupo que recibió el suplemento estratégico incrementó este indicador en $29 \%$. Además, los ingresos percibidos por la venta de leche y los índices evaluados no presentaron diferencia entre tratamientos $(\mathrm{p}<0.05)$.

En la literatura se encuentran pocos trabajos que incluyen la evaluación económica de la suplementación estratégica en sistemas de lechería en trópico alto colombiano. Entre estos, Avellaneda et al. (2018) indicaron que la implementación de diversas estrategias de suplementación estratégica formuladas a mínimo costo y con concentraciones bajas de proteína cruda disminuyeron los costos de suplementación entre 16 y $31 \%$ en cuatro fincas ubicadas en las principales cuencas lecheras. Desafortunadamente, en este trabajo no se incluyó la evaluación de otros indicadores económicos. Finalmente, la incorporación de suplementos elaborados en el predio conduce a una mayor gestión (formulación de la dieta, compra de materias primas y mezclado de la dieta) por parte de los productores. En este sentido, cada sistema de producción deben definir si la implementación de esta estratégia promueve una mayor rentabilidad del sistema de producción.

\section{Conclusión}

La inclusión de suplementos estratégicos en dietas de vacas lecheras no modificó la producción y la composición láctea, ni los indicadores económicos evaluados. Sin embargo, el costo del suplemento estratégico fue $37.5 \%$ menor que el suplemento comercial, pudiéndose constituir en una estrategia para reducir los costos de alimentación sin afectar los parámetros productivos del sistema de producción lechero.

\section{Literatura Citada}

1. Álvarez HJ, Dichio L, Larripa M. 2007. Suplementación energética en vacas con distintos niveles de producción de leche y asignación de pastura. Rev Argen Produc Anim 27: 151-157.

2. A.O.A.C. Association of Official Agricultural Chemists. 2005. Official methods of analysis. Washington DC, USA.

3. Avellaneda Y, Mancipe E, Vargas JJ. 2018. Suplementación a mínimo costo con baja concentración de proteína para vacas lecheras del trópico alto colombiano. En: XXVI Reunión de Asociación Latinoamericana de Producción Animal (ALPA). Guayaquil, Ecuador.

4. Correa HJ, Pabón ML, Carulla JE. 2008. Valor nutricional del pasto kikuyo (Pennisetum clandestinum Hoechsst Ex Chiov) para la producción de leche en Colombia (Una revisión): I - Composición química y degestibilidad ruminal y posruminal. Livest Res Rural Dev 20(59). [Internet]. Disponible en: http:// www.lrrd.org/lrrd20/4/corra20059.htm

5. FEDEGAN-SENA. 2013. Costos modales en ganadería de leche. Trópico alto de Colombia: ventana a la competitividad ganadera. Bogotá, Colombia: FEDEGAN. 110 p.

6. Garg MR, Sherasia PL, Bhanderi BM, Phondba BT, Shelke SK, Makkar HPS. 2013. Effects of feeding nutritionally balanced rations on animal productivity, feed conversión efficiency, feed nitrogen use efficiency, rumen microbial protein supply, parasitic load, immunity and enteric methane emissions of milking animals under field conditions. Anim Feed Sci Technol 179: 24-35.

7. [IDEAM] Instituto de Hidrología, Meteorología y Estudios Ambientales. 2018. Reporte meteorológico. Bogotá, Colombia. 
8. López S. 2005. In vitro and in situ techniques for estimating digestibility. In: Dijkstra J, Forbes JM, France J (eds). Quantitative aspects of ruminant digestion and metabolism. $2^{\text {nd }}$ ed. Wallingford, UK: CABI Publishing. $\mathrm{p} 87-121$.

9. Macdonald K, Roche J. 2011. Body condition scoring made easy, the official field guide. $3^{\text {rd }}$ ed. Auckland, New Zealand: Dairy NZ. 43 p.

10. Martínez R, Martínez, Martínez MV. 2011. Diseño de experimentos en ciencias agropecuarias y biológicas con SAS, SPSS, R y Statistix. Bogotá, Colombia: Ed. Fondo Nacional Universitario. $803 \mathrm{p}$.

11. Makkar H. 2016. Smart livestock feeding strategies for harvesting triple gain - the desire outcomes in planet, people and profit dimensions: a developing country perspective. Anim Prod Sci 56: 519-534. doi: 10.1071/AN15557

12. [NRC] National Research Council. 2001. Nutrient requirements of dairy science. $7^{\text {th }}$ ed. Washington, USA.

13. Perea C, Garcés YJ, Muñoz LS, Hoyos JL, Gómez JA. 2018. Valoración económica del uso de ensilaje de residuos piscícolas en la alimentación de Oreochromis spp biotechnol. Sector Agropec Agroind 16: 43-51.

14. Pulido JI. 2005. Caracterización de los sistemas de producción de leche del trópico de altura en los departamentos de Boyacá y Cundinamarca. Informe Técnico Final. Corporación Colombiana de Investigación Agropecuaria (CORPOICA). Bogotá, Colombia. 112 p.

15. Rao I, Peters M, Castro A, SchultzeKraft R, White D, Fisher M, Miles J, et al. 2015. Livestock plus - the sustainable intensification of foragebased agricultural systems to improve livelihoods and ecosystems services in the tropics. Trop Grasslands 3: 59-82. doi: 10.17138/TGFT(3)59-82

16. Roche JR, Berry DP, Bryant AM Burke CR, Butler ST, Dillon PG, Donaghy DJ, et al. 2017. A 100 year review: a century of change in temperate grazing dairy systems. J Dairy Sci 100: 10189-10233. doi: $10.3168 / j \mathrm{js} .2017-13182$

17. [SAS] Statistical Analysis System. 2017. Online Doc 9.4. SAS Institute Inc. Carolina del Norte, USA.

18. Tarazona AM, Ceballo MC, Naranjo $J F$, Cuartas $C A$. 2012. Factor affecting forage intake and selectivity in ruminants. Rev Colomb Cienc Pec 25: 473-487.

19. Tilley JMA, Terry RA. 1963. A twostage technique for the in vitro digestion of forage crops. Grass Forage Sci 18: 104-111. doi: 10.1111/j.1365-2494.1963.tb00335. $\mathrm{x}$

20. Valencia D. 2011. Determinación de producción forrajera para el ajuste de cargas animales en sistemas de rotación en la hacienda Los Alpes utilizando como método un botanal. Tesis de Industrial Pesquero. Caldas, Colombia: Corporación Universitaria Lasallista. $67 \mathrm{p}$.

21. Van Soest PJ, Robertson JB, Lewis BA. 1991. Methods for dietary fiber, neutral fiber and no starch polysaccharides in relation to nutrition. J Dairy Sci 74: 35833597. doi: 10.3168/jds.S0022-0302(91)78551-2

22. Vargas JJ, Sierra AM, Mancipe EA, Avellaneda Y. 2018. El kikuyo, una gramínea presente en los sistemas de rumiantes en trópico alto colombiano. Rev CES Med Zootec 13: 137-156.

23. Vargas J, Pabón M, Carulla J. 2014. Producción de metano in vitro en mezclas de gramíneas y leguminosas del trópico alto colombiano. Arch Zootec 63: 397-407. doi: 10.4321/S0004-05922014000300001 